Neue Formen der Politikberatung

\title{
Entscheidungsbezogene Wissenschaft?
}

\author{
Die Gesellschaft, insbesondere die Politik, \\ stellt neue Ansprüche an die Wissenschaft. \\ Obwohl sie nicht alle Erwartungen erfüllen \\ kann, hat die Wissenschaft als Berater der \\ Politik auch ihre Berechtigung. \\ Von Gotthard Bechmann
}

D as Verhältnis von Gesellschaft und Wissenschaft hat sich in den letzten Jahrzehnten verändert. Das erkenntnisorientierte und explanatorische Selbstverständnis der Wissenschaft als Ort praxisferner Kontemplation, Experimentierkunst und Theoriebildung, wie es dem Ideal der klassischen Physik entsprach und von dort seinen Siegeszug antrat, ist heute nur noch in Teilen der Wissenschaft anzutreffen.

Die gegenwärtigen Diskussionen um die gesellschaftliche Funktion von Forschung werden von unterschiedlichen, teilweise auch widersprüchlichen Erwartungen geprägt. Forschung soll nicht nur gesichertes Wissen für die Gesellschaft bereitstellen, sondern auch zur Lösung von gesellschaftlichen Problemen durch die Produktion neuen Wissens beitragen. Die stärkere Einbindung von Forschung in ihre gesellschaftlichen Kontexte und die Forderung nach ihrem praktischen Nutzen sind Symptome des Wandels von Forschung und bieten gleichzeitig den Ausgangspunkt der wissenschaftlichen Reflexion über ihr Verhältnis zur Gesellschaft.

Auch die Debatte um das Entstehen der Wissensgesellschaft, die die alte Industriegesellschaft ablösen soll, und der Streit um neue Formen der Wissensproduktion, die den Übergang von einer wesentlich akademisch geprägten Wissenschaft, Mode 1 genannt, zu einer stärker gesellschaftlichen integrierten Wissenschaft, Mode 2 genannt, repräsentieren, sind Ausdruck des Wandels von Forschung.

\section{Bedeutungswandel der Forschung}

Der Wandel der gesellschaftlichen Funktion von Forschung kündigt sich in einer Reihe von unterschiedlichen Entwicklungen an. Heute wird wissenschaftliches Wissen in vielen gesellschaftlichen Bereichen von der Wirtschaft über die Politik bis hin zur Erziehung erzeugt, die nicht unmittelbar in Beziehung zum traditionellen Wissenschaftssystem stehen. Beispiele da- für finden sich in Industrielaboren, staatlich geförderten und anwendungsbezogenen Instituten oder auch außeruniversitären, privaten Forschungseinrichtungen. Darüber hinaus werden nicht mehr nur disziplinär sozialisierte Wissenschaftler, sondern auch die so genannten Abnehmer, der „Laie“ oder Auftraggeber, in die Erzeugung wissenschaftlichen Wissens mit einbezogen.

Indem Forschung zunehmend in Wirtschaft, Politik und Kultur nachgefragt wird und in diesen Systemen Leistungen übernimmt, gewinnt der Verwendungskontext wissenschaftlichen Wissens an Bedeutung. Diese Entwicklung überschreitet die wissenschaftstheoretische Position, die den Entdeckungszusammenhang (context of discovery) und den Rechtfertigungszusammenhang (context of justification) streng unterscheidet. Wenn Entdeckungs- und Rechtfertigungskontext zusammenfallen, dann ist auch Forschung nicht mehr allein in der Lage, das Wissen und seine Verwendungsweisen (Expertisen) mithilfe seiner eigenen Qualitätsstandards und Evaluationsmechanismen wie beispielsweise Peer Review zu kontrollieren. Mit der Kontextualisierung von Forschung verändert sich auch ihr Legitimationsmodus. Für die Glaubwürdigkeit wissenschaftlichen Wissens wird nicht nur die „Objektivität“, sondern auch der praktische Nutzen für seine Anwender in den unterschiedlichen Anwendungsbereichen bedeutend.

Durch die Erweiterung und Verschmelzung der Bewertungskontexte kommt es zur Neubewertung wissenschaftlicher Funktionsweisen und Leistungspotenziale, der zufolge auch Grundlagenforschung - gewollt oder ungewollt - gesellschaftlich relevant sein soll und auf der reinen Wissensproblematik übergeordnete Problemstellungen ausgerichtet werden kann. Die Produktion wissenschaftlichen Wissens soll unmittelbar in die wirtschaftlichen und politischen Entscheidungsprozesse integriert werden. Damit wächst der Stellenwert der Wissenschaft für die Ökonomie als Impuls für Innovationen sowie für die Politik als Lieferant von Themen, Problemen und Entscheidungswissen. Wissenschaft steigert damit die Handlungskapazität der gesellschaftlichen Bereiche, indem sie nicht nur Erklärungen, sondern auch Modelle zur Wirklichkeitsgestaltung und Entscheidungsalternativen entwirft.

\section{Wissenschaft im politischen Entscheidungsprozess}

Was aber bedeutet diese Entwicklung für die Arrangements der Wissenschaft? Mit der Herausbildung einer „knowledge based industry" und dem Entstehen einer staatlich geförderten 
und strategisch-programmatisch ausgerichteten gesellschaftlichen Vorsorgeforschung entstehen neue Wissensformen, die sich zum einen in ihrer Organisationsweise nicht mehr der klassischen Trias von Grundlagenforschung, angewandter Forschung und Kommerzialisierung fügen. Zum anderen wird wissenschaftlich gewonnenes Erfahrungswissen durch sie in kontrolliertes Entscheidungswissen transformiert.

Die Entstehung einer entscheidungsbezogenen Wissenschaft kann man als Antwort des Wissenschaftssystems auf die neuen Anforderungen betrachten, die vonseiten der Gesellschaft an die Wissenschaft gestellt werden.

Wissenschaft übernimmt damit die Aufgabe, politische Ziele zu erreichen und soziale Bedürfnisse zu befriedigen. Wissenschaftliche Analyse und Konstruktion praktischer Zusammenhänge (Vorsorge- und Risikoforschung), prognostischer Leistungen sind dabei eingeschlossen, werden zunehmend für politische und soziale Innovationen benötigt, nachdem sich das Sekundärfolgesyndrom (nicht intendierte Folgen) zu einer unübersehbaren Größe ausgeweitet hat.

Der politische Entscheidungsprozess stellt sich zunehmend als ein Zusammenspiel von Wissenschaftlern, professionellen Interessenvertretern, Verwaltungsfachleuten und Politikern dar. Die letzte Entscheidungsmacht bleibt zwar immer noch dem Politiker vorbehalten, der Wissenschaftler erlangt allerdings zunehmend Definitionsmacht und Lösungskompetenz. Die gut dreißig Jahre anhaltende gesellschaftliche Thematisierung der ökologischen Problematik bedeutet für die Wissenschaft zweierlei: Zum einen ergeben sich für sie neue Tätigkeitsfelder, die interdisziplinäre Zusammenarbeit erfordern. Es werden neue Themen kreiert und nicht zuletzt große Mengen von Fördermitteln erschlossen. Zum anderen steigt der Bedarf an wissenschaftlicher Beratungskapazität. Nicht nur die Politik, auch Unternehmen und Verbände, sofern sie sich mit Umweltpolitik und ökologischer Regulierung befassen, müssen auf wissenschaftlich erzeugtes Wissen zurückgreifen.
Indem aber die Wissenschaft die neue Rolle übernommen hat, sieht sie sich mit zwei Problemen konfrontiert, die das traditionelle Selbstverständnis der Wissenschaft, rational und wertfrei zu sein, infrage stellen:

I Sie stößt in Anwendungsbereiche vor, in denen sogar diejenigen Interdependenzen, die sich noch erfassen oder gar technisch erzeugen lassen, nicht mehr beherrschbar sind. Im Unterschied zur „normal science“, wo sich die Wissenschaft nur solche Fragen stellt, die sie mit ihren Mitteln beantworten kann, muss man heute erkennen, dass die Wissenschaft an nachweisbare Grenzen ihrer Analyse- und Prognosefähigkeit stößt. Die Grenzen der Wissenschaft zeigen sich besonders deutlich auf dem Gebiet der Umweltforschung. In neuartiger Weise entsteht gewusstes Nichtwissen.

- Mit der Integration in den politischen Regulierungsprozess verliert die Wissenschaft ihre Unschuld, die sie durch die Norm der Wertfreiheit so lange propagandistisch verteidigt hat. Wertfreiheit bedeutet unter anderem auch Objektivität der Erkenntnis. Was wissenschaftlich gewusst wird, ist bis auf weiteres, das heißt bis zur Widerlegung, unbezweifelbares Wissen, das für alle gilt. Der Konsens der Wissenschaftsgemeinde ist hier das Kriterium. Genau dies lässt sich in den neuen Anwendungsbereichen nicht mehr aufrechterhalten. Wissen, obwohl von Wissenschaftlern produziert, mit wissenschaftlichen Methoden erzeugt, stellt sich schnell als kontextgebunden, als unsystematisch gewonnen, als schnell revisionsbedürftig und vor allem als selektiv heraus. Mit anderen Worten: Es ist umstritten.

\section{Wissen und Politikberatung}

Die damit verbundenen Probleme aktualisieren sich gegenwärtig vor allem in der wissenschaftlichen Politikberatung, weil hier wissenschaftliches Wissen in der sozialen Dimension als Legitimationsressource für die Begründung wie auch als Infor-

\section{politische ökologie \\ Die Zeitschrift für Querdenkerinnen und Vordenker}

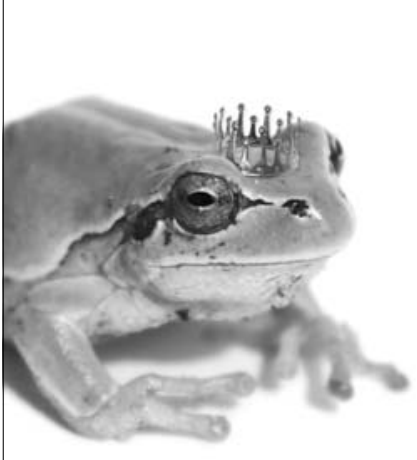

\section{Zukunft wachküssen \\ Leitlinien für ein nachhaltiges Regierungsprogramm}

Renommierte Expertinnen und Experten treten für ein Ende der Discount-Kommunen ein, erklären die historische Chance in der EU-Chemikalienpolitik und fordern ein Ende der ministerialen Kleinstaaterei. Die politische ökologie ${ }^{97-98}$ wagt einen Sprung nach vorne: überzeugend, konkret und machbar.

_Wofür spielt die Couleur einer Regierung noch eine Rolle?

_Braucht ein sozial und ökologisch zukunftsfähiger Welthandel neue Leitplanken?

_Was können wir von den Außerirdischen lernen?

Mit Beiträgen von M. Jänicke, H. E. Ott, S. Thomas, U. Brand, U. Kallee, A. Grunwald, U. Röhr, T. Korbun u.v.m.

_Stillen Sie Ihre Neugier!_Fordern Sie die politische ökologie 97-98 an_18,- EUR (inkl. Versand)/28,80 CHF!

_ISBN 3-86581-004-7_oekom verlag_Fax +49/(0)81 91/970 00-103_neugier@oekom.de_www.oekom.de

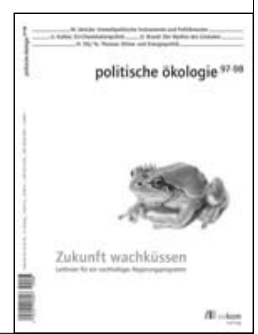


mationsressource zur Orientierung von Entscheidungen benötigt wird. Wissenschaftliche Erklärungsangebote spielen im politischen Kontext eine signifikante Rolle, da sie über eine heuristische Funktion hinsichtlich des generellen Ausmaßes, der Struktur und der wahrscheinlichen Richtung des globalen Wandels verfügen. Sie bilden somit die kognitive Grundlage der öffentlichen und politischen Kontroverse. Die Art und Weise, wie Probleme wahrgenommen und gedeutet werden, strukturiert auch das Terrain für Konflikte. Forschung erweist sich in hohem Maße als politisch relevant, gerade weil sie sich als die Instanz etabliert hat, die Ursachen und Folgen von globalen Veränderungen zurechnet, beispielsweise Gewinner und Verlierer des Klimawandels identifiziert, Verantwortliche und Betroffene benennt sowie Ziele und Strategien der politischen Problembewältigung aufzeigt.

Für den Verlauf der politischen Kontroverse gewinnen Prozesse der wissenschaftlichen Zurechnung eine herausragende Bedeutung, da die Definition von Kausalrelationen und entsprechenden Ursache-Wirkungsmechanismen auch die Verteilung von Verantwortung und Ressourcen folgenreich verändert. Im Fall Klimawandel wurde die wissenschaftliche Diskussion in dem Moment politisch relevant, als Forschung begann, Veränderungen in der natürlichen Umwelt auf menschliche Aktivitäten zurückzuführen. Dieser Wandel der Zurechnung erweist sich als politisch außerordentlich bedeutsam, da er die Problematik des Klimawandels von einer naturgegebenen Gefahr in ein entscheidungsabhängiges Risiko transformiert und auf diese Weise einen politischen Handlungsraum konstituiert.

\section{Zusätzliche Risiken}

Zu diesen neuen Funktionen, die der Wissenschaft zum Teil aufgedrängt, zum Teil von ihr freiwillig übernommen worden, kommen auf dem Gebiet der Ökologie und der Technikfolgen noch zusätzliche Risiken hinzu. Meist entstammen die Probleme, zu deren Lösung die Wissenschaft um Rat gefragt wird, nicht ihrer eigenen theorie- oder empiriegesteuerten Erfahrung. Sie sind weder im disziplinären Zusammenhang der Wissenschaft noch in dem aktuellen Forschungsstand verankert, sondern verdanken sich fremden Handlungsproblemen, auf die das Wissen erst zugeschnitten werden muss

Dies gilt auch dann, wenn das Thema, wie etwa der Klimawandel, erst durch die Wissenschaft „entdeckt“ und wesentlich mitgeformt wird. Auch in diesem Fall bleibt die Art der Thematisierung nicht in der ausschließlichen Regie des wissenschaftlichen Diskurses. Gleichwohl nimmt die Bedeutung der wissenschaftlichen Expertise zu, weil sie trotz aller Unsicherheit der Wissensproduktion der einzige legitime Weg ist, empirisch gestütztes Wissen zu erzeugen, das allgemein anerkannt ist und umgesetzt werden kann. Ihre Funktion für die Politik liegt dann aber nicht mehr so sehr in der Verkündung sicheren Wissens, sondern besteht, wenn man es pointiert ausdrückt, in einem qualitativ hochwertigen Management von Unsicherheit. Kern dieser Sichtweise ist die
Kommunikation über die Unsicherheit und Revidierbarkeit der eigenen Wissensproduktion im Austausch mit Öffentlichkeit und Politik.

\section{Management von Unsicherheit}

Der Begriff Management von Unsicherheit macht auf die zeitliche Struktur des Wissens aufmerksam. Phänomene wie Klimawandel, Grundwasserverschmutzung oder Verkarstung sind zum einen Resultate von Handlungen, unter anderem auch von wissenschaftlichem Handeln. Zum anderen hängen sie in ihrer Erfahrbarkeit und Sichtbarkeit von den Beobachtungen und Modellierungen der Wissenschaft ab, so dass sie als zeitlicher Bestandteil der Wissensstruktur analysierbar werden. Globale Probleme existieren nicht einfach außerhalb dieses Zusammenhangs, sondern werden durch die Wissenschaft definiert, organisiert und im öffentlichen Diskurs mediatisiert.

Das, was wir über diese Phänomene wissen, hängt somit auch von der Gestaltbarkeit und Organisationsweise der Forschung ab, die wiederum auf externe Ressourcen und Unterstützung angewiesen ist. Dieser konstitutive Zusammenhang von Wissen und Handeln mit einer im Prinzip unendlich zeitlichen Perspektive hat natürlich Rückwirkungen auf die Organisationsweise der Forschung, die im Rahmen der Politikberatung relevant wird. Hier werden neue Formen der Wissenserzeugung und Wissensvermittlung gesucht.

Betrifft die Beobachtung über die sich herausbildenden Ansätze eines Integrated Assessment die Seite der Forschungsorganisation und hier insbesondere Fragen der Bündelung disziplinübergreifenden Wissens, so zeigen sich auf den gesellschaftlichen Problemfeldern, die durch hohe Unsicherheit und einen starken Bedarf an (natur)wissenschaftlich-technischen Wissen gekennzeichnet sind, eine weitere interessante Entwicklung, die die kommunikativen Voraussetzungen der Wissenschaft betont: die Herausbildung von Epistemic Communities, zu Deutsch epistemischen Gemeinschaften.

Epistemic Communities sind professionelle Wissensgemeinschaften, die gleichermaßen Wissens- und Akteurssysteme sind. Sie bestehen aus hoch spezialisierten Experten, die sich in genau definierten Problemfeldern auskennen und hohe Kompetenz besitzen. Sie verfügen über ein Verständnis von Kausalprozessen und die entsprechende Methodik, Wissen zu erzeugen und in strategische Anweisungen zu überführen. Sie werden von den politischen Entscheidungsträgern und der Gesellschaft als Vermittler legitimen Wissens anerkannt. Ihre Aufgabe ist es, wissenschaftlich fundierte Deutungen und Konstruktionen zur Verfügung zu stellen, die in den Politikprozess übersetzt und dort abgearbeitet werden können.

\section{Argumente statt Konflikte}

Ihr Bezugssystem wird nicht von konfligierenden politischen Interessen bestimmt, sondern durch wissenschaftlich argumentative Auffassungen von den zu verhandelnden Sachver- 
halten. Die Einheit der Communities ist in der Gemeinsamkeit von Problemdefinition, Kausalhypothese und normativen Annahmen über Handlungsbedarf und Handlungsoptionen begründet. Sie besteht, in anderen Worten, im Konsens über ein Set wissenschaftlicher und normativer Ideen, nicht unähnlich einem wissenschaftlichen Paradigma im Sinne von Kuhn. Im Beziehungsgeflecht der internationalen Politik treten solche "networks of knowledge-based-experts“ im Zusammenhang der Technologie- und Umweltpolitik auf.

Ihre Wissensbasis ist durch eine Summe von rein technischen Informationen sowie durch Theorie und Interpretation bestimmt. Zusätzlich muss über das reine Faktenwissen hinaus noch die Fähigkeit vorhanden sein, trotz Wissenslücken, Unsicherheiten und Nichtwissen Konsens zu erzeugen. Erst in der Form dieses „konsensualen Wissens" findet das Expertenwissen Eingang in die Politik und trägt dort zur Problemdefinition bei. Epistemic Communities koppeln auf der institutionellen Ebene Prozesse wissenschaftlicher Forschung an politische Programmformulierung. Damit werden Anschlüsse zwischen Politik und Wissenschaft erzeugt, die es ermöglichen, in einem dauerhaften kommunikativen Prozess jeweils Programmentwicklung (Handeln) und Forschung (Wissen) in einer dynamischen $\mathrm{Zu}$ sammensetzung auf Dauer zu stabilisieren.

\section{Wissenschaftliche Beratung hat Bedeutung}

Seinen Wert als Beratung gewinnt dieses Vorgehen trotzdem durch die Versicherung, dass

I alle zum Zeitpunkt der Veröffentlichung verfügbaren Informationen methodisch einwandfrei berücksichtigt werden und

I sowohl alternative wissenschaftliche Ansätze als auch andere nichtwissenschaftliche Thematisierungen der wissenschaftlichen Reflexion unterliegen.

Damit macht sich die wissenschaftliche Politikberatung anschlussfähig zugleich für weitere wissenschaftliche Forschung wie für die gesellschaftliche Kommunikation. In der Iteration zwischen diesen beiden Referenzen liegt ihre Funktion bei der Abarbeitung gesellschaftlicher Unsicherheit. Die beratende Funktion bleibt dann trotz des Wahrheitsverlustes insofern in Kraft, als es keine in ihrer Transparenz und Zurechenbarkeit verlässlichere Auskunft gibt. In dieser Art etwa kann man eine glaubwürdige Funktion der wissenschaftlichen Politikberatung formulieren, nachdem die Wahrheitsfiktion ihre Glaubwürdigkeit verloren hat.

\section{Literatur}

Bechmann, G.: Risk and the Postmodern Society. In: Zucker, B. (Hrsg.): RisikoDialog - Von der Idee zur konkreten Umsetzung. Institut für Versicherungswirtschaft der Universität St. Gallen. St. Gallen 2001, S. 139-154.

Bechmann, G.: Das Konzept der „Nachhaltigen Entwicklung“ als problemorientierte Forschung - Zum Verhältnis von Normativität und Kognition in der Umweltforschung. In: Brand, K.-W. (Hrsg.): Nachhaltige Entwicklung und Transdisziplinarität. Angewandte Umweltforschung Bd. 16. Berlin 2000, S. 31-46.

Bogner, A./ Torgersen, H. (Hrsg): Wozu Experten. Ambivalenzen der Beziehung von Wissenschaft und Politik. Wiesbaden 2005.

Lahhusen, C.: Kontraktuelle Politik. Politische Vergesellschaftung am Beispiel der Luftreinhaltung in Deutschland, Frankreich, Großbritannien und den USA. Weilerswist 2003.

Maasen,S./ Weingart,P. (Hrsg.): Democratization of Expertise? Exploring Novel Forms of Scientific Advise in Political Decision Making. Dordrecht 2005.

Nowotny, H. et al.: Re-Thinking Science. Knowledge and the Public in an Age of Uncertainty. Cambridge 2001

Stehr, N. : Wissenspolitik. Frankfurt/M. 2003.

Weingart, P.: Die Stunde der Wahrheit? Zum Verhältnis der Wissenschaft zu Politik, Wirtschaft und Medien in der Wissensgesellschaft. Weilerswist 2001.
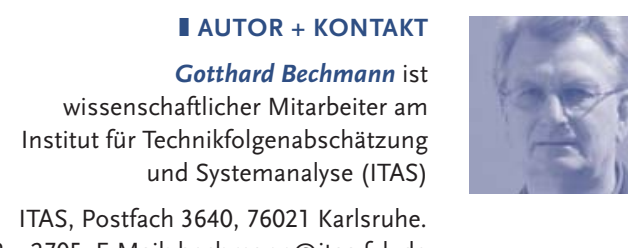

Tel. 07247/ 82 - 2705, E-Mail: bechmann@itas.fzk.de

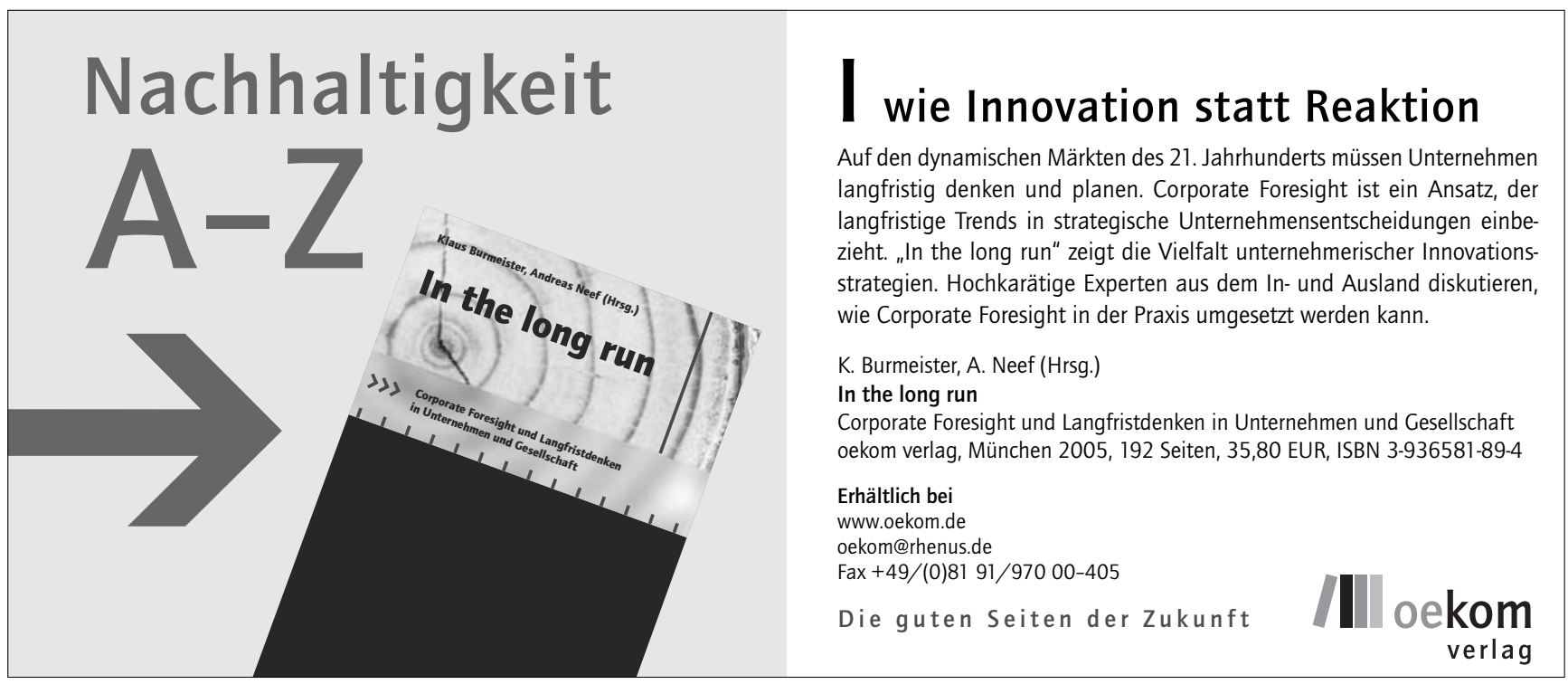


(c) 20I0 Authors; licensee IÖW and oekom verlag. This is an article distributed under the terms of the Creative Commons Attribution Non-Commercial No Derivates License (http://creativecommons.org/licenses/by-nc-nd/3.o/), which permits unrestricted use, distribution, and reproduction in any medium, provided the original work is properly cited. 